\title{
210.
}

\section{ON THE CUBIC TRANSFORMATION OF AN ELLIPTIC FUNCTION.}

[From the Philosophical Magazine, vol. xv. (1858), pp. 363-364.]

LET

$$
z=\frac{\left(a^{\prime}, b^{\prime}, c^{\prime}, d^{\prime} \chi x, 1\right)^{3}}{\left(a, b, c, d \gamma(x, 1)^{3}\right.}
$$

be any cubic fraction whatever of $x$, then it is always possible to find quartic functions of $z, x$ respectively, such that

$$
\frac{d z}{\sqrt{\left(\mathrm{a}, \mathrm{b}, \mathrm{c}, \mathrm{d}, \mathrm{e} \gamma(z, 1)^{4}\right.}}=\frac{d x}{\sqrt{\left(A, B, C, D, E_{\gamma}(x, 1)^{4}\right.}} .
$$

This depends upon the following theorem, viz. putting for shortness,

$$
\begin{aligned}
& U=\left(a, b, c, d \gamma(x, y)^{3},\right. \\
& U^{\prime}=\left(a^{\prime}, b^{\prime}, c^{\prime}, d^{\prime} \gamma(x, y)^{3},\right.
\end{aligned}
$$

and representing by the notation

$$
\text { disct. }\left(a U^{\prime}-a^{\prime} U, \quad b U^{\prime}-b^{\prime} U, \quad c U^{\prime}-c^{\prime} U, \quad d U^{\prime}-d^{\prime} U\right) \text {; }
$$

or more shortly by

$$
\text { disct. }\left(a U^{\prime}-a^{\prime} U, \ldots\right) \text {, }
$$

the discriminant in regard to the facients $(\lambda, \mu)$ of the cubic function

$$
\left(a U^{\prime}-a^{\prime} U, \quad b U^{\prime}-b^{\prime} U, \quad c U^{\prime}-c^{\prime} U, \quad d U^{\prime}-d^{\prime} U \gamma \lambda, \mu\right)^{3} \text {; }
$$

or what is the same thing, the cubic function

$$
\begin{array}{r}
(a, b, c, d \gamma \lambda, \mu)^{3} \cdot\left(a^{\prime}, b^{\prime}, c^{\prime}, d^{\prime} \chi x, y\right)^{3} \\
-\left(a^{\prime}, b^{\prime}, c^{\prime}, d^{\prime} \chi \lambda, \mu\right)^{3} \cdot(a, b, c, d \gamma x, y)^{3} ;
\end{array}
$$

and by $J\left(U, U^{\prime}\right)$ the functional determinant, or Jacobian, of the two cubics $U, U^{\prime}$; the theorem is that the discriminant contains as a factor the square of the Jacobian, or that we have

$$
\text { disct. }\left(a U^{\prime}-a^{\prime} U, \ldots\right)=\left\{J\left(U, U^{\prime}\right)\right\}^{2} \cdot\left(A, B, C, D, E \gamma(x, y)^{4} .\right.
$$


For assuming this to be the case, then (disregarding a mere numerical factor) we have

$$
U d U^{\prime}-U^{\prime} d U=J\left(U, U^{\prime}\right)(y d x-x d y),
$$

and the two equations give

$$
\frac{U d U^{\prime}-U^{\prime} d U}{\sqrt{\operatorname{disct}\left(a U^{\prime}-a^{\prime} U, \ldots\right)}}=\frac{y d x-x d y}{\sqrt{\left(A, B, C, D, E \gamma(x, y)^{4}\right.}},
$$

whence writing $z$ for $U^{\prime} \div U$, and putting $y$ equal to unity, we have

$$
\frac{d z}{\sqrt{\text { disct. }\left(a z-a^{\prime}, \ldots\right)}}=\frac{d x}{\sqrt{\left(A, B, C, D, E \gamma(x, 1)^{4}\right.}}
$$

where disct. $\left(a z-a^{\prime}, \ldots\right)$, or at full length,

$$
\text { disct. }\left(a z-a^{\prime}, b z-b^{\prime}, c z-c^{\prime}, d z-d^{\prime}\right) \text {, }
$$

is a given quartic function of $z$,

$$
=\left(\mathrm{a}, \mathrm{b}, \mathrm{c}, \mathrm{d}, \mathrm{e} \gamma(z, 1)^{4}\right.
$$

suppose; and this proves the theorem of transformation.

The assumed subsidiary theorem may be thus proved: suppose that the parameter $\theta$ is determined so that the cubic

$$
U+\theta U^{\prime}
$$

may have a square factor, the cubic may be written

$$
\left(a+\theta a^{\prime}, b+\theta b^{\prime}, c+\theta c^{\prime}, d+\theta d^{\prime} \gamma x, y\right)^{3},
$$

and the requisite condition is

$$
\text { disct. }\left(a+\theta a^{\prime}, \ldots\right)=0 \text {; }
$$

there are consequently four roots; and calling these $\theta_{1}, \theta_{2}, \theta_{3}, \theta_{4}$, we have identically

$$
\text { disct. }\left(a+\theta a^{\prime}, \ldots\right)=K\left(\theta-\theta_{1}\right)\left(\theta-\theta_{2}\right)\left(\theta-\theta_{3}\right)\left(\theta-\theta_{4}\right) \text {, }
$$

or what is the same thing,

$$
\text { disct. }\left(a U^{\prime}-a^{\prime} U, \ldots\right)=K\left(U+\theta_{1} U^{\prime}\right)\left(U+\theta_{2} U^{\prime}\right)\left(U+\theta_{3} U^{\prime}\right)\left(U+\theta_{4} U^{\prime}\right) \text {. }
$$

Now any double factor of $U$ or $U^{\prime}$ (that is the linear factor which enters twice into $U$ or $\left.U^{\prime}\right)$ is a simple factor of $J\left(U, U^{\prime}\right)$, and we have $J\left(U, U^{\prime}\right)=J\left(U, U+\theta U^{\prime}\right)$, and consequently

$$
J\left(U, U^{\prime}\right)=J\left(U, U+\theta_{1} U^{\prime}\right)=\& c \text {; }
$$

hence the double factors of each of the expressions $U+\theta_{1} U^{\prime}, U+\theta_{2} U^{\prime}, U+\theta_{3} U^{\prime}$, $U+\theta_{4} U^{\prime}$ are simple factors of $J\left(U, U^{\prime}\right)$, or what is the same thing, $J\left(U, U^{\prime}\right)$ is the product of four linear factors, which are respectively double factors of the product

$$
\left(U+\theta_{1} U^{\prime}\right)\left(U+\theta_{2} U^{\prime}\right)\left(U+\theta_{3} U^{\prime}\right)\left(U+\theta_{4} U^{\prime}\right)
$$

or this product contains the factor $\left\{J\left(U, U^{\prime}\right)\right\}^{2}$, which proves the theorem.

2, Stone Buildings, W.C., March 5, 1858. 